\title{
Adaptive wireless network multi-objective optimization algorithm based on image synthesis
}

\author{
Jianwei Zhang ${ }^{1 *}$ and Xueya Zhang ${ }^{2}$
}

\begin{abstract}
Multi-objective optimization problems can be divided into continuous multi-objective optimization problems and discrete multi-objective optimization problems, and discrete multi-objective optimization is not universal. In practical applications, there are many discrete multi-objective optimization problems. The solution of different problems needs to design different evolutionary multi-objective algorithms according to their specific conditions. The threshold in the traditional multi-objective optimization of wireless network is a preset constant. It has relatively poor performance in the synthesis of images with different noise levels. An adaptive wireless network multi-objective optimization algorithm based on image synthesis is proposed. Based on the maximum inter class variance and maximum peak signal to noise ratio (SNR), an adaptive wireless network multi-objective optimization algorithm is established. The accuracy and noise immunity of image synthesis are also considered. In order to avoid the effect of threshold increase on algorithm efficiency, multi-objective optimization algorithm is introduced into the algorithm. Experiments show that the method proposed in this paper is accurate and robust and has good universality for the synthesis of different noise images.
\end{abstract}

Keywords: Image synthesis, Adaptive, Wireless network, Multi-objective optimization

\section{Background}

Image synthesis plays an important role in the field of computer vision and the pattern recognition [1]. At present, the mainstream image synthesis methods include cluster synthesis method, morphological synthesis method, region synthesis method, and threshold synthesis method [2]. In the multiple synthesis methods, the threshold synthesis method has been widely applied in the image processing field due to its simplicity and effectiveness [3], in which, the maximum between-class variance method is one of the most common and important synthesis methods. The maximum between-class variance method only takes the gray value information of the pixel into consideration while ignoring its spatial correlation; hence, the algorithm has relatively poor noise immunity $[4,5]$. To solve this issue, the two-dimensional Otsu method is put forward by introducing the mean value of the pixel neighborhood, which has enhanced the noise

\footnotetext{
* Correspondence: wenguzhixin980@163.com

${ }^{1}$ College of Physics and Optoelectronic Technology, Baoji University of Arts and Sciences, Baoji 721016, China

Full list of author information is available at the end of the article
}

immunity of the algorithm to some extent. On the basis of the two-dimensional Otsu method, the neighborhood median value is further taken into consideration. And a kind of three-dimensional Otsu method is put forward [6]. Both the two-dimensional and the three-dimensional Otsu methods have enhanced the noise resistance capacity of the algorithm to a certain extent. However, the complexity of the algorithm has been greatly increased, and the efficiency of the algorithm is relatively low. In order to improve the efficiency of the three-dimensional Otsu method, scholars both at home and abroad have conducted extensive research [7, 8]. A kind of fast recursive method for the three-dimensional Otsu method is put forward by establishing a look-up table, which, however, is implemented at the expense of the memory space. The three-dimensional Otsu method is converted into one-dimensional Otsu method three times, which can improve the efficiency of the algorithm without extra memory consumption. However, the direct finite difference method is adopted to divide the three-dimensional histogram, which takes only the two regions adjacent to the main diagonal into consideration. This may often lead to 
inaccurate synthesis [9]. In this regard, the three-dimensional histogram is transformed into a one-dimensional image projection histogram to improve the efficiency of the algorithm on the premise of taking the entire histogram region into consideration. In addition, a kind of threshold post-processing strategy is adopted to correct the noise and enhance the noise resistance capacity of the algorithm. Although the multi-objective optimization algorithm has relatively good synthesis performance, the threshold value in the post-processing process is a preset constant, and the synthesis effect depends heavily on the image itself; hence, the algorithm generally has relatively poor universality [10].

This paper first makes a deep understanding of the traditional algorithm of image fusion. In this regard, the threshold value in the multi-objective optimization process of the adaptive wireless network is taken as a variable. And the accuracy of the algorithm synthesis is measured by the variance between classes. The peak signal to noise ratio (hereinafter referred to as PSNR (peak signal to noise ratio) for short) is used to measure the noise immunity performance of the algorithm. And a kind of adaptive wireless network multi-objective optimization algorithm that takes both the accuracy and the noise immunity into consideration is established in this paper. Given that the multi-objective optimization algorithm has shown relatively good performance in the aspect of threshold synthesis, the multi-objective optimization algorithm is introduced in this paper to put forward a kind of adaptive wireless network multi-objective optimization algorithm (hereinafter referred to as AWNMMOA for short) based on the image synthesis, to avoid the reduction in the efficiency of the algorithm due to the increase in the threshold value. The test results show that the method put forward in this paper has strong noise immunity and good robustness and can properly synthesize the images that contain different noise conditions.

\section{A brief introduction to the principle of image fusion}

Recently, image fusion has been widely used in many fields, such as military weapons, machine vision, remote sensing telemetry, medical image processing, and automatic target recognition system. The application of image fusion in the military field is mainly focused on the target acquisition of weapons such as precision-guided missiles and unmanned attack aircraft. Because these weapons need to lock the target in the execution of strategic bombing and they have many uncertain factors in high speed flight, such as weather and obstacles, it is not easy to produce very successful photographs in one shot, so it needs to be fused to multisensory or multi-time image data to get a clearer post fusion image. The intelligent system on the weapon will interpret the image and the target image in the database which are stored in the database. If the feature information is similar, the attack launch command is executed. If the feature information is not similar, then the data will be processed. The quality of image fusion is closely related to the guidance precision of intelligent-guided weapons, and because the speed of the missile is very fast, the speed of image fusion processing is also higher. Human recognition and understanding of the target is a process of multi-source information fusion. Human vision, hearing, taste, and so on are integrated to identify a target, and then compare and match the cognitive experience of their own brain, and finally decide. For example, two eyes of human beings are equivalent to two sensors, which are fused around each other and transmit information to the central nervous system. In the development of intelligent robot technology, one of the most important aspects is machine vision, especially the principle of image fusion. In medical analysis, image fusion is also very important, for example, CT images show more of the human skeleton, while MRI images are more detailed in the details of the muscles and ligaments of the human body. The fusion of these two kinds of images will be more conducive to the accurate grasp of the patient's condition. In the field of target recognition, image fusion is also very important, such as the fusion of color information of concentrated energy, such as the recognition of the edge, texture, and details of the target, to get a clearer recognition of the target object and so on. The application area of image fusion is also important in resource survey, landform mapping, and crop growth prediction.

Image fusion ring refers to the process of using a mathematical model to integrate multiple images from multiple sensors into one image. This image is used to meet specific application requirements. In the whole fusion project, the advantages of different sensor images are effectively combined to selectively remove redundant information. According to the different stages of image fusion processing, it is divided into pixel level, feature level, and decision level. Different levels of fusion have different algorithms and scope of application. In the three levels of image fusion, pixel level fusion is the basis of image fusion at all levels. This method preserves the initial information of the source image as much as possible and provides quite rich, accurate, and reliable information for the fusion of other layers, which is beneficial to further analysis of the fused image, so as to form the optimal decision and fusion. Plan Fig. 1 shows the basic block diagram of pixel level image fusion process. 


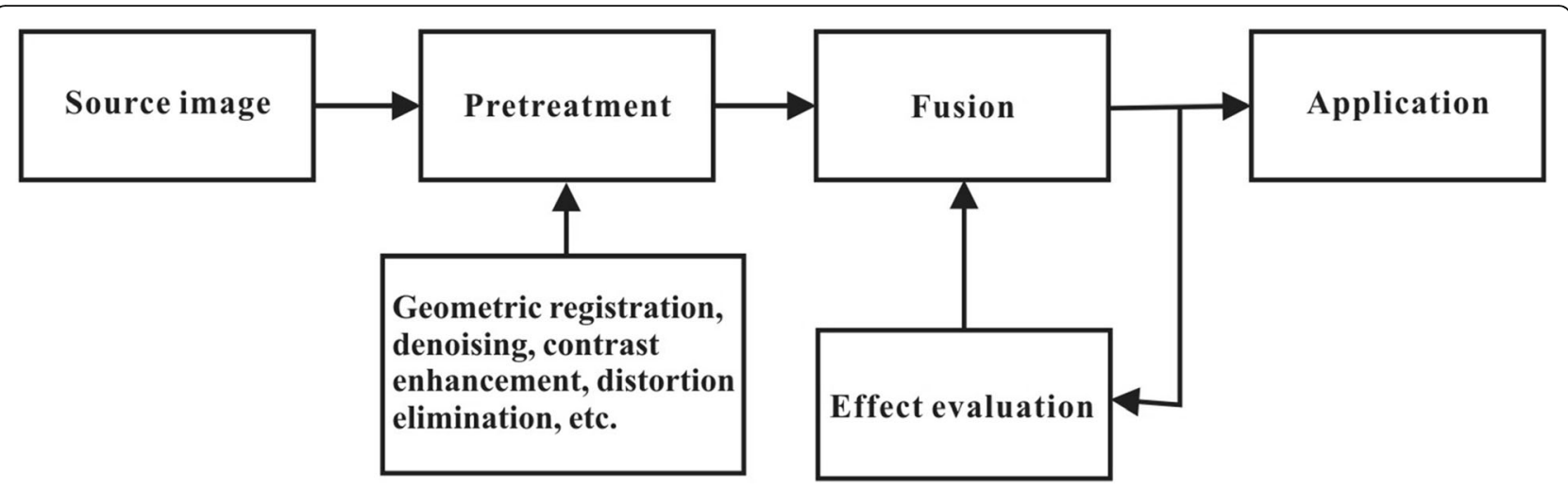

Fig. 1 Basic principle block diagram of pixel level fusion

The purpose of image fusion is to deal with the complementary information of the multi-source image, so that the clarity of the image is improved, and the redundancy data provided by the multi-sensor is processed to enhance the reliability of the image. Image fusion is part of multi-sensor information fusion. In practical terms, the two have many common points. In fact, multi-source information fusion technology has biological basis. Multi-source information fusion is embodied in human and animal's ability to enhance survival probability, which is used reasonably and naturally to perceive the living environment and identify dangerous skills. It is like that human beings can naturally integrate all kinds of information (such as sound, image, smell, and heat) from many sensors (organs) of the human body (such as sound, image, taste, and heat) to integrate a large number of existing knowledge, recollection, experience, etc. stored in the human brain. The application of image fusion technology is very wide, so the research of image fusion is of great significance. The more

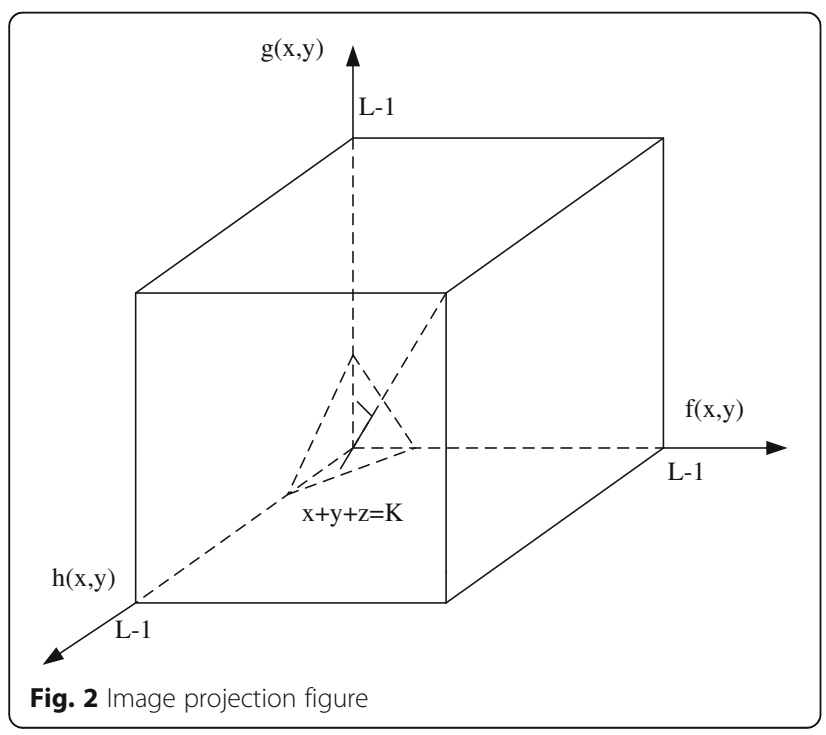

mature fusion algorithm and the more efficient and effective fusion system will greatly promote the development of image-related technology.

\section{Method-adaptive wireless network multi- objective optimization algorithm}

In view of the low efficiency, poor precision, difficulty in extension, and other defects of the three-dimensional Otsu method, the pixel points in the three-dimensional histogram are first projected to the main diagonal. In addition, the one-dimensional image projection histogram is established in accordance with the frequency of the projection point (denoted as $p_{r}$ ). (The projection process is shown in Fig. 2 as follows.)

Based on the image projection histogram, a kind of new maximum variance rule between the classes is established as follow:

$$
\sigma_{B}^{2}(T)=P_{0}(T)\left[\mu_{0}(T)-\mu_{T}\right]^{2}+P_{1}(T)\left[\mu_{1}(T)-\mu_{T}\right]^{2}
$$

in which, $P_{0}(T)=\sum_{r=0}^{T} p_{r}, P_{1}(T)=\sum_{r=T+1}^{\sqrt{3}(L-1)} p_{r}, \mu_{0}(T)$ $=\frac{\sum_{r=0}^{T} r p_{r}}{P_{0}(T)}, \mu_{1}(T)=\frac{\sum_{r=T+1}^{\sqrt{3}(L-1)} r p_{r}}{1-P_{0}(T)}, \mu_{T}=\sum_{r=0}^{\sqrt{3}(L-1)} r p_{r}$.

To enhance the noise resistance performance of the algorithm, a kind of post-processing strategy based on the threshold value is designed. The basic idea is that if most of the pixels in the pixel neighborhood fall in a certain class, the pixels are classified into this class. And the treatment process is as follows:

Step 1 The threshold value is set as $Q=s, s \in[0.5,1]$.

Step 2 For the pixel $(x, y)$, the ratio of pixels $P_{B}$ and $P_{O}$ in the eight neighborhood that belong to the background and the target respectively is calculated. It is assumed that the $n_{B}$ and $n_{O}$ stand for the number of pixels in the pixel 8 neighborhood that belong to the background and the target, respectively. 
Then, the calculation process is shown in Eqs. (2) and (3) as follows:

$$
\begin{aligned}
& P_{B}=\frac{n_{B}}{n_{O}+n_{B}}=\frac{n_{B}}{8} \\
& P_{O}=\frac{n_{O}}{n_{O}+n_{B}}=\frac{n_{B}}{8} 1-P_{B}
\end{aligned}
$$

Step 3 The size of $P_{B}$ and $P_{O}$ is compared to that of $Q$, respectively. If $\left.P_{B}\right\rangle Q$, it indicates that most of the pixels about the pixel $(x, y)$ belong to the background, and this pixel should also be classified as the background at this point. On the contrary, if $\left.P_{O}\right\rangle Q$, the pixel should be classified as the target. In case neither conditions above are met, it indicates that the numbers of the pixels that belong to the background and target about the pixel $(x, y)$ are close. At this point, it is considered as an edge pixel, and its classification remains unchanged.

Step 4 In order to make binary wavelet transform to realize the multi-scale decomposition of signal and reconstruct the original signal, the low pass filter and bandpass filter in BWT must satisfy the constraint conditions. The constraint conditions guarantee the low pass filtering characteristic of the scale function and the bandpass filtering characteristic of the wavelet function. The complete reconstruction constraint guarantees the signal to be passed over inverting and undistortionless reconstruction. It is determined whether the pixels in the binary image have been processed. If the processing has not been completed, return to step 2 and continue the execution; otherwise, the processing result is output.

Although the image projection histogram obtained from the three-dimensional histogram has certain noise immunity, the selection of the threshold value is based only on the maximum between-class variance criteria, and the objective is to pursue the accuracy of the image synthesis. Although the post-processing strategy based on the threshold value can correct the noise to a certain extent, the value taken for the threshold depends heavily on the image itself. Figure 3a gives Lena images with the noise strengths of 0 (that I, not containing noise), 0.05 , 0.10 , and 0.15 , respectively, to facilitate the analysis of the effect of the noise on the threshold value $Q$. The resultant image obtained after the synthesis is locally enlarged so that it can be recognized by the human eye. And the enlargement process is shown in Fig. $2 b$ to Fig. 2e. From Fig. 2e, it can be seen that when the noise intensity is 0 , there is no noise in the 8 neighborhoods of the pixel. And it is not necessary to change the pixel classification at this time, that is, the threshold value $Q$ $=1.0$ is the most suitable; when the noise intensity is 0.05 , there are 1 noise point and 7 non-noise points in the 8 neighborhoods of the pixel as pointed by the arrow. At this time, the pixel should be corrected as a non-noise point, that is, the threshold value $Q=6 / 8=$ 0.75 is the most suitable. When the noise intensity is 0.10 , there are 2 noise points and 6 non-noise points in the 8-neighborhood of the pixel pointed by the arrow. As is known from the above, the threshold value $Q=5 / 8=0.625$ is the most suitable. When the noise intensity is 0.15 , there are 3 noise points and 5 non-noise points in the 8 neighborhoods of the pixel pointed by the arrow. Currently, the threshold value $Q=4 / 8=0.5$ is the most suitable. The analysis process shows that the reasonable value taken for the threshold value is closely related to the noise contained in the image. And the fixed value of $Q$ set as 0.7129 is a general value obtained through many tests, which is not the optimal value of all the images. Hence, it can be seen that the universality of the multi-objective optimization algorithm is relatively poor.

The peak signal to noise ratio is widely used to evaluate the performance of the filter algorithm. And in view of the relatively poor universality of the multi-objective optimization algorithm, the threshold value $Q$ is taken as a variable, and the maximum peak signal to noise ratio is taken as the objective to determine the adaptive value taken for the threshold value $Q$. Hence, the peak signal to noise ratio is as follows:

$$
\operatorname{PSNR}(Q)=20 \lg \left(\frac{255}{\sqrt{\frac{\sum_{i=1}^{M} \sum_{j=1}^{N}(I(i, j)-\hat{I}(i, j))^{2}}{M \times N}}}\right)
$$

In which, $I$ stands for the reference image (for the images with the known prior knowledge, the artificial synthesis results can be used as the reference image; considering the universality of the algorithm, the result image after the filtering of the original image is used as the reference image in this paper), $\hat{I}$ stands for the synthesis result which is obtained in accordance with the threshold value $Q . M \times N$ stands for the image size. Equation (4) indicates that the greater the similarity between the synthesis result and the reference image, the larger the peak signal to noise ratio is, and the stronger the noise immunity performance of the algorithm is.

The synthesis threshold value $T$ and the post-processing threshold value $Q$ are combined into the threshold vector $(T, Q)$. A kind of adaptive wireless network multi-objective optimization algorithm that can take both the accuracy and the noise immunity into 


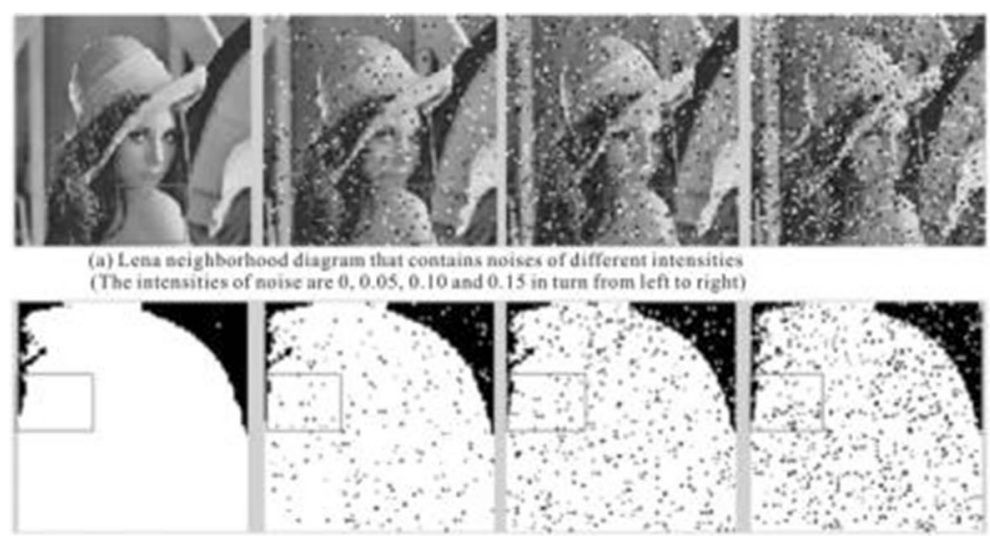

(b) Enlarged vies of the syntbesis result of the figure (a)

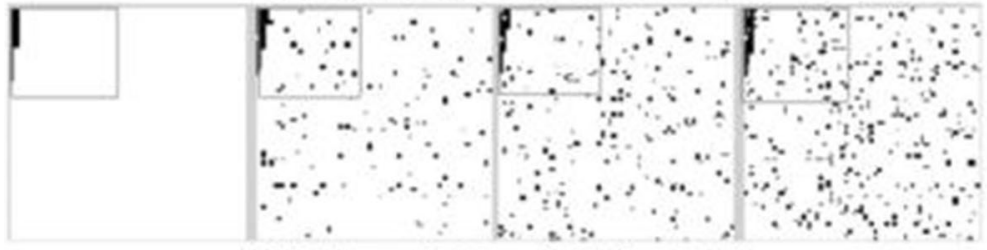

(c) Enlarged view of the figure (b)

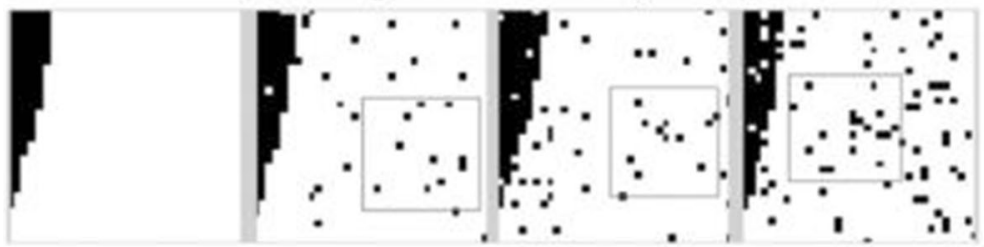

(d) Enlarged view of the figure (c)

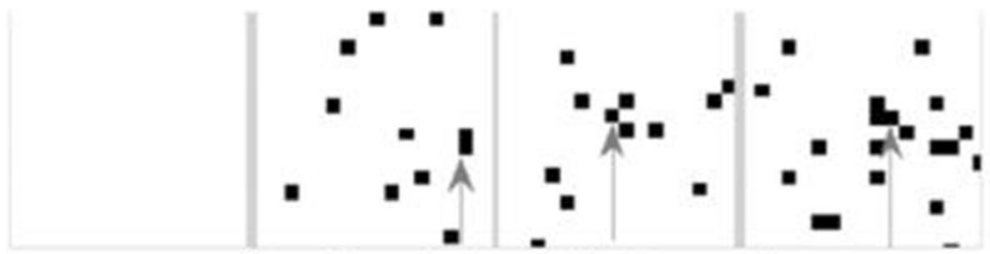

(e) Enlarged view of the figure (d)

Fig. 3 a-e Lena neighborhood diagram containing noises of different intensities

consideration into consideration is established based on the combination of the maximum between-class variance criterion as shown in Eq. (1) and the peak signal to noise ratio criterion as shown in the Eq. (4) as follows:

$$
\left.\begin{array}{c}
\max g(T, Q)=\left\{\begin{array}{l}
g_{1}(T)=\sigma_{B}^{2}(T) \\
g_{2}(Q)=\operatorname{PSNR}(Q)
\end{array}\right\} \\
\text { s.t.T }=1,2, \cdots, \sqrt{3}(L-1) Q \in[0.5,1.0]
\end{array}\right\}
$$

From Eq. (1) and the post-processing based on the threshold value, it can be known that the search space for the threshold value $T$ and $Q$ is $\sqrt{3} L$ and $\left(n_{O}+n_{B}\right) / 2$, respectively. Hence, it can be seen from Eq. (4) that for any threshold value $\left(T^{*}, Q^{*}\right)$ that is determined arbitrarily, it is necessary to carry out the operation $M \times N$ times to calculate the peak signal to noise ratio. Hence, the algorithm complexity of the adaptive multi-objective optimization algorithm for wireless network as shown in Eq. (5) is $O\left(\sqrt{3} L \times \frac{n_{O}+n_{B}}{2} \times M \times N\right)$, while the complexity of the traditional multi-objective optimization algorithm is $\sqrt{3} L$. This indicates that although the multi-objective optimization algorithm of the adaptive wireless network put forward in this paper can take the accuracy and the noise immunity of the image synthesis into consideration at the same time, the complexity of the algorithm is increased greatly, which has seriously affected the efficiency of the algorithm. 


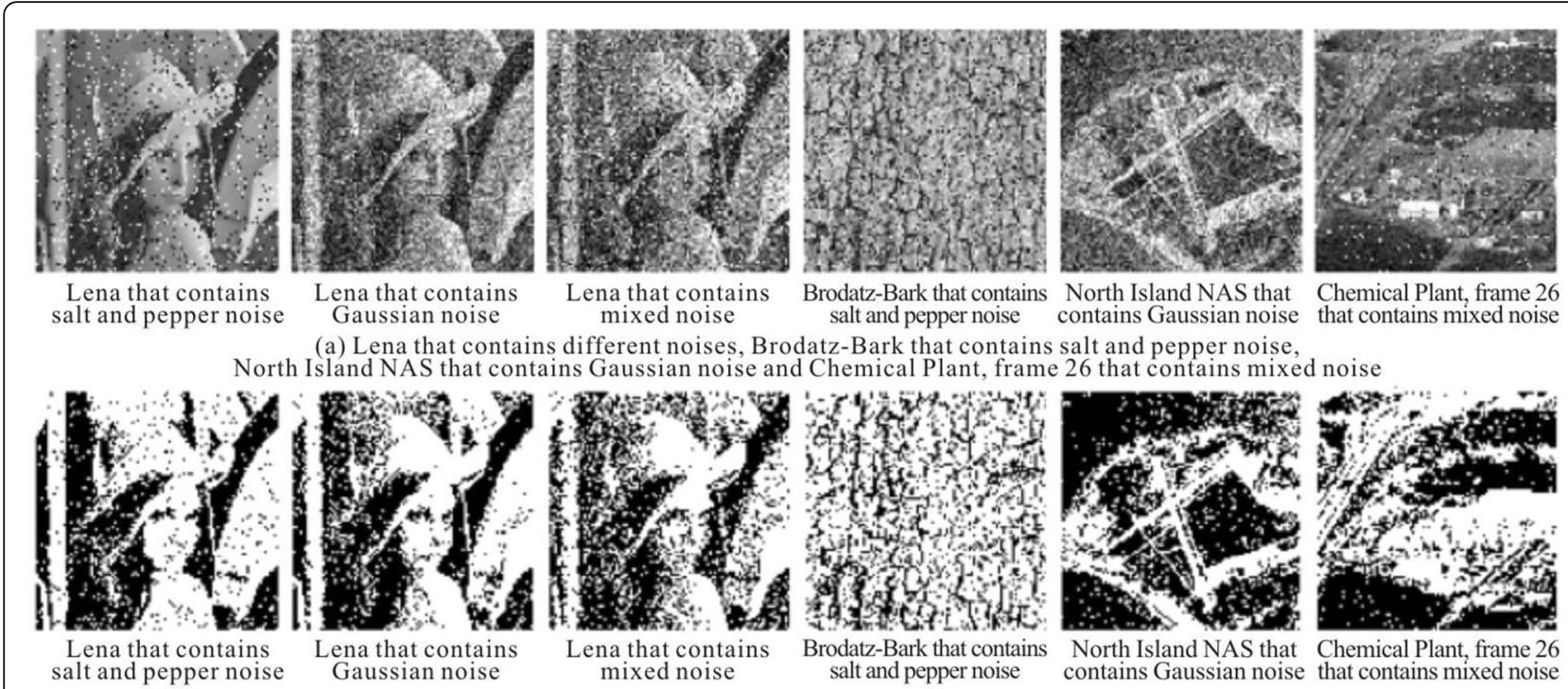

(b) Segmentation result of the traditional 3-dimensional Otsu method

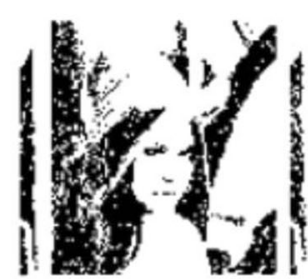

Lena that contains salt and pepper noise

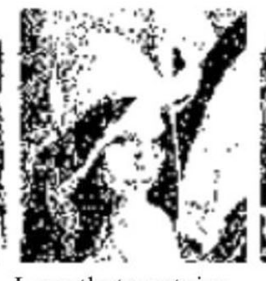

Lena that contains

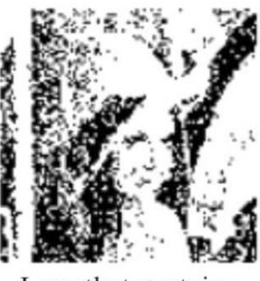

Lena that contains mixed noise
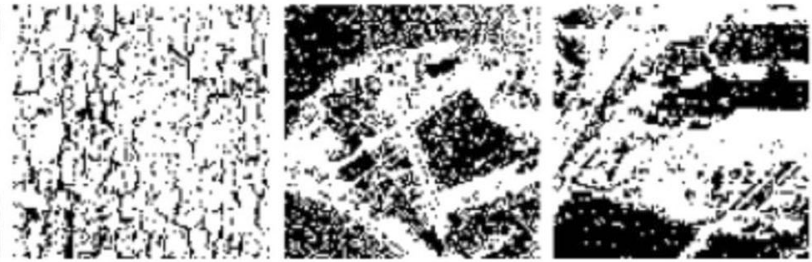

Brodatz-Bark that contains North Island NAS that Chemical Plant, frame 26 salt and pepper noise contains Gaussian noise that contains mixed noise

(C) Segmentation result of the 3-dimensional Otsu method based on decomposition

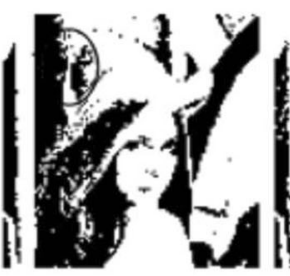

Lena that contains salt and pepper noise

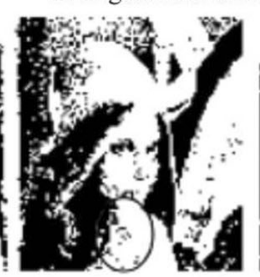

Lena that contains Gaussian noise

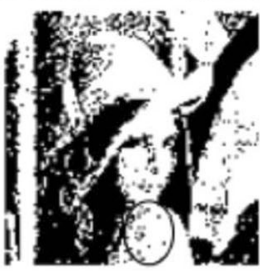

Lena that contains mixed noise

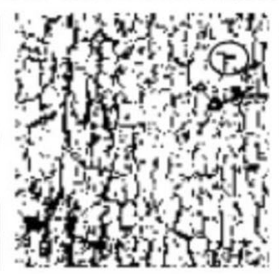

Brodatz-Bark that contains salt and pepper noise
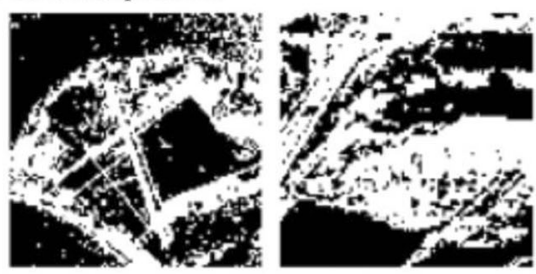

North Island NAS that Chemical Plant, frame 26 contains Gaussian noise that contains mixed noise

(d) Segmentation result of the cross sectional projection Otsu method

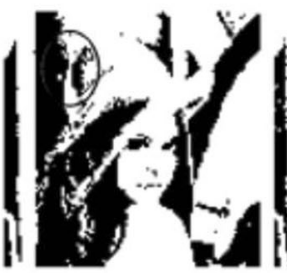

Lena that contains salt and pepper noise

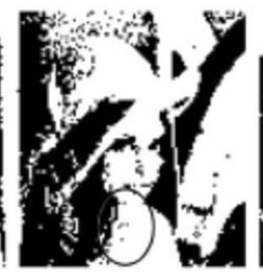

Lena that contains Gaussian noise

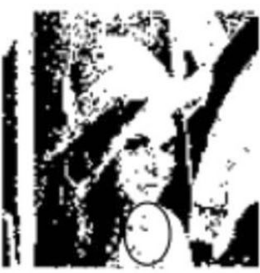

Lena that contains mixed noise

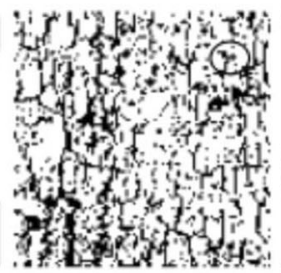

Brodatz-Bark that contains salt and pepper noise
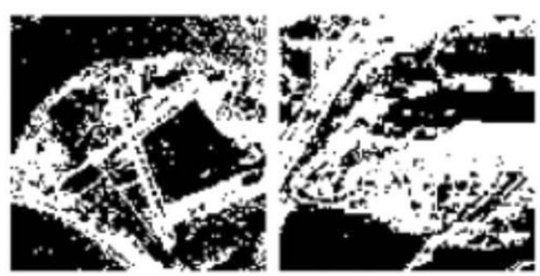

North Island NAS that Chemical Plant, frame 26 contains Gaussian noise that contains mixed noise

(e) Segmentation result of the multi-objective cross sectional projection Otsu method put forward in this paper

Fig. 4 a-e Comparison of the synthesis results of each algorithm

4 Result and discussion: experimental testing and analysis

In order to test the performance of the synthesis algorithm put forward in this paper, the following two groups of experiments are carried out: (1) test of the effectiveness of the adaptive wireless network multi-objective optimization algorithm, which is compared to the three-dimensional Otsu method in the 


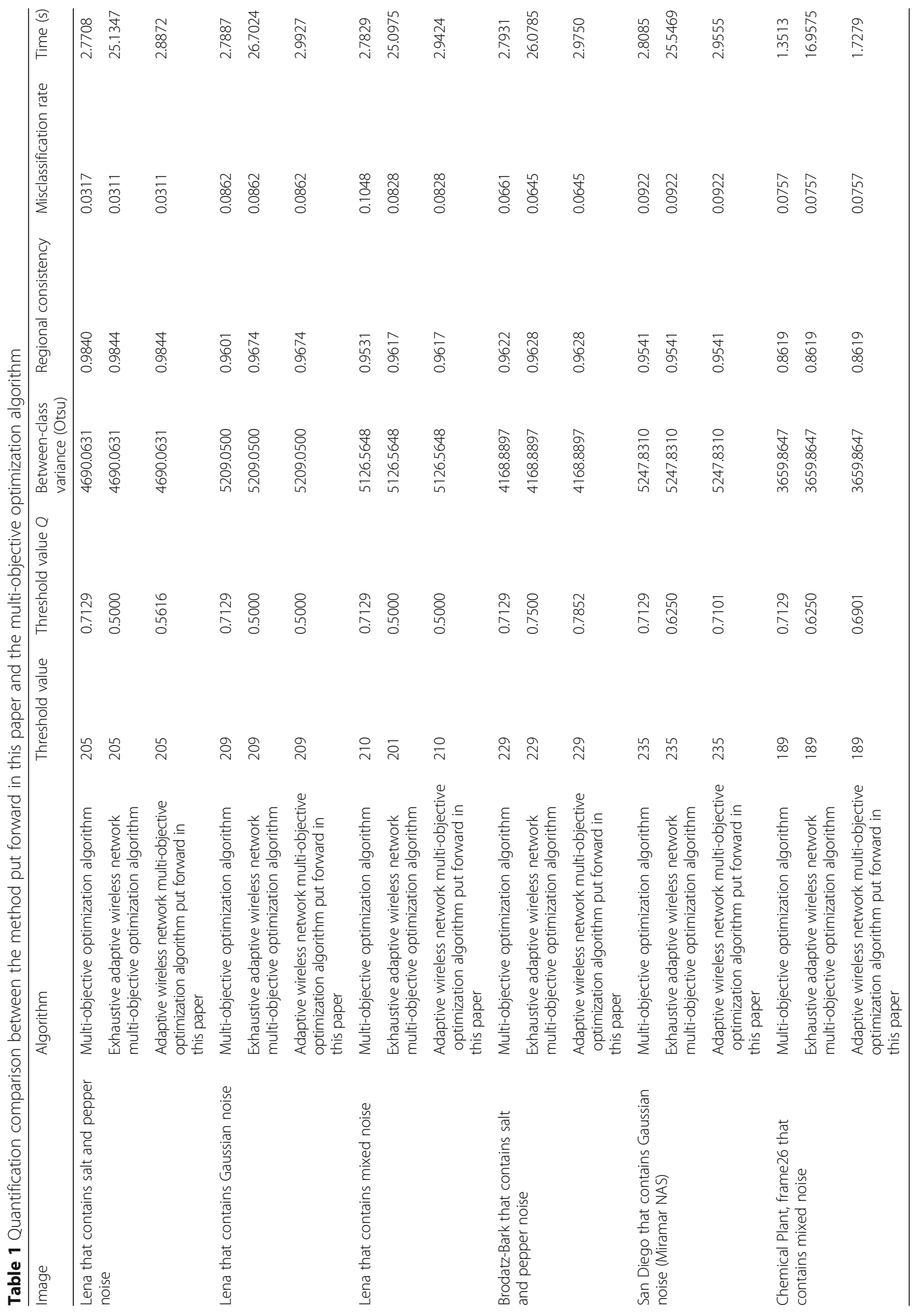


same class, to verify the effectiveness of the adaptive wireless network multi-objective optimization algorithm put forward in this paper; (2) test of the robustness of the algorithm. The changes in the performance of each algorithm when the intensity of the noise is continuously increasing are compared to analyze the robustness of the algorithm in the noise resistance performance. The experimental operating environment is as follows: Pentium (R) $2.7 \mathrm{GHz}$ CPU and $2 \mathrm{G}$ memory, and the programming language is MATLABR2009b.

\subsection{Verification of the validation of the synthesis model}

It is judged whether the processing of the pixels in the binary image is completed to verify the effectiveness of the adaptive multi-objective wireless network optimization algorithm put forward in the paper. And it is compared with the traditional three-dimensional Otsu method, the three-dimensional Otsu method based on decomposition and the adaptive wireless network multi-objective optimization algorithm. In the experiment, the Lena images that contain the salt and pepper noise, the Gaussian noise, and the mixed noise; the Brodatz-Bark images that contain the salt and pepper noise; the San Diego (Miramar NAS) images that contain the Gaussian noise; and the Chemical Plant, frame26 images that contain the mixed noise (as shown in Fig. 4a) are selected as the test objects. The population size $N$ and the maximum number of iterations $m_{\mathrm{it}}$ of the AWNMMOA are set to 10 and 20, respectively. The weight $\lambda$ is set to 0.5 . And the other parameters are set as the same as the above. From Fig. 3b to Fig. 3e, it can be seen that the traditional three-dimensional Otsu method makes use of the direct finite difference method to divide the histogram and determine the optimal threshold value by the approximate calculation. Therefore, the synthesis error is relatively large. The three-dimensional Otsu method has improved the accuracy of the image synthesis to a certain extent as the approximate calculation of the traditional three-dimensional Otsu method is avoided. The multi-objective optimization algorithm and the adaptive wireless network multi-objective optimization algorithm are all based on the image projection histogram to avoid the defects of the direct finite difference method. In addition, the post-processing strategy based on the threshold value is adopted to correct the noise. Hence, the synthesis effect of the two is relatively good and close. The circle mark part in the Lena image shows that the multi-objective Otsu method for the adaptive wireless network put forward in this paper has stronger noise resistance performance. And the circle mark part in the Brodatz-Bark image shows that the adaptive wireless network multi-objective optimization algorithm put forward in this paper can better maintain the texture information of the image. The threshold value $Q$ of the adaptive wireless network multi-objective optimization algorithm put forward in this paper is used as a variable, which has stronger universality for the synthesis of the images with different noise conditions.

The synthesis effects of the multi-objective optimization algorithm and the adaptive multi-objective optimization algorithm put forward in this paper are not easy to be distinguished visually. Therefore, the threshold value $T$, the threshold value $Q$, the regional consistency, the error resolution rate, the calculation time, and other indexes are selected to carry out quantitative comparison of the two. In order to verify the effectiveness of the AWNMMOA in improving the efficiency of the algorithm, the exhaustive adaptive wireless network multi-objective optimization algorithm is further used as the comparison object. From Table 1, as the multi-objective optimization algorithm and the adaptive wireless network multi-objective optimization algorithm determine the synthesis threshold value $T$

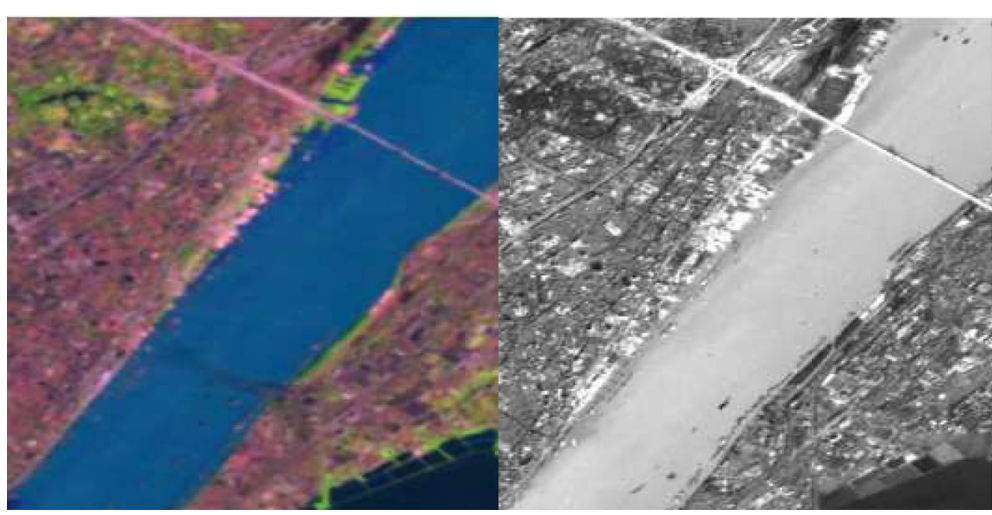

(a) MS image

(b) PAN image

source diagram

Fig. 5 a, $\mathbf{b}$ Source diagram 


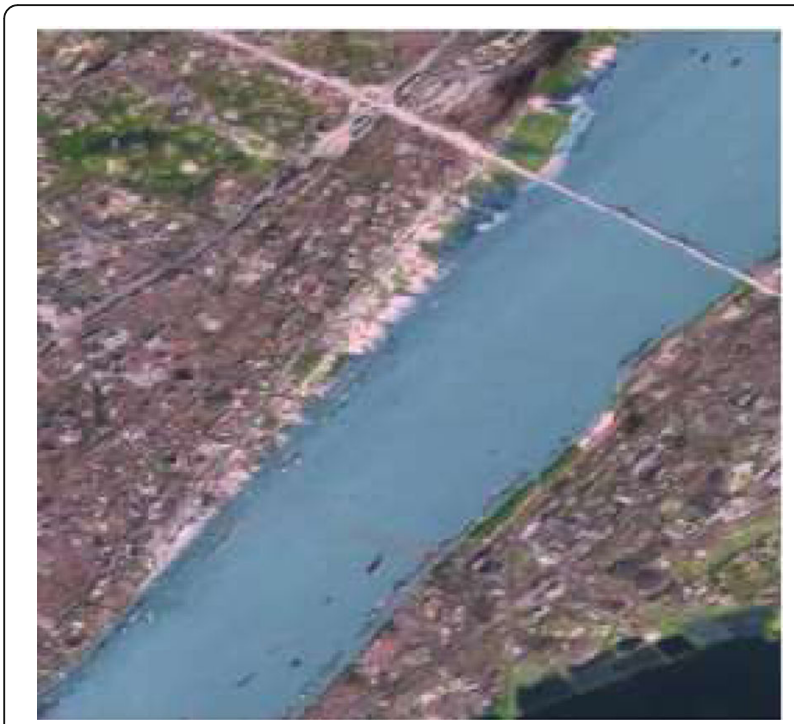

fusion image effect

Fig. 6 Fusion image effect

based on the image projection histogram and the maximum between-class variance criteria, the threshold value $T$ of the three methods and the variance between the classes are equal. For the Lena images that contain different noises and the Brodaz-Bark images that contain the salt and pepper noise, the multi-objective optimization algorithm for the adaptive wireless network has greater regional consistency than that of the multi-objective optimization algorithm. In addition, the pixel error resolution rate is smaller than that of the multi-objective optimization algorithm. This indicates that the adaptive post-processing threshold value $Q$ in this paper can better correct the noise. For the San Diego (Miramar NAS) image that contains the Gaussian noise and the Chemical Plant, frame 26 image that contains the mixed noise, the threshold value $Q$ of both the multi-objective optimization algorithm and the adaptive wireless network multi-objective optimization algorithm put forward in this paper is in the interval of $(5 / 8,6 / 8)$. In this paper, the synthesis effect of the adaptive wireless network multi-objective optimization algorithm is the same as that of the exhaustion method. However, the algorithm efficiency is greatly improved by adopting the image synthesis to solve the optimal threshold value. The quantitative comparison result is basically consistent with the qualitative analysis result as mentioned above. And it has strongly verified again from the quantitative perspective the effectiveness of the synthesis method put forward in this paper.

\subsection{Analysis of the robustness of the algorithm}

The AWNMMOA algorithm is proposed in this paper and shows better performance in algorithm test and image preprocessing de noising. The algorithm is used in pixel level image fusion, and compared with other classical algorithms, better experimental results are obtained. The subjective and objective evaluation indexes of the fused image have been improved accordingly. In this paper, the fusion of MS and PAN images and the fusion of multi-focus images are selected. In the process and framework of integration, it is based on traditional methods. The source image is shown in Fig. 5 where a is a MS image and b is a PAN image. In this paper, the AWNMMOA algorithm

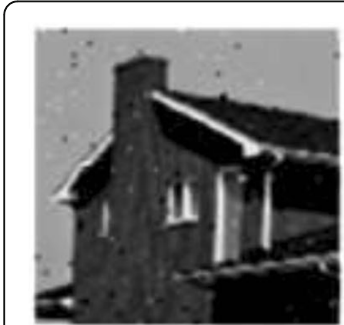

$\delta=0,0]$

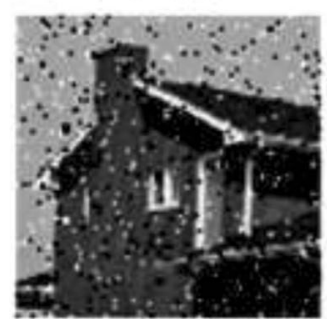

$\delta=0.06$

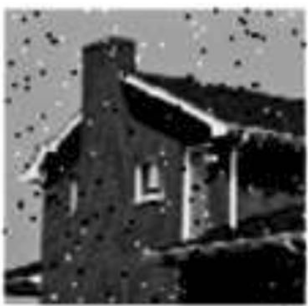

$\delta=0.02$

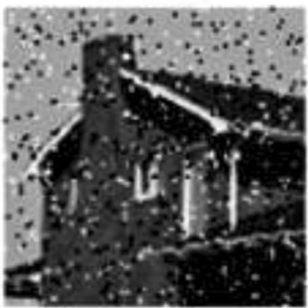

$\delta=0.07$

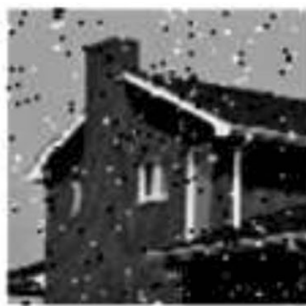

$\delta=0.03$

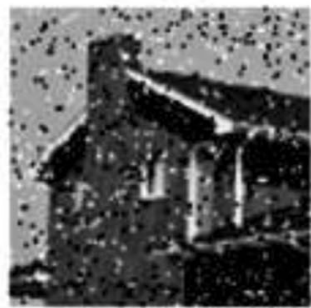

$\delta=0.08$

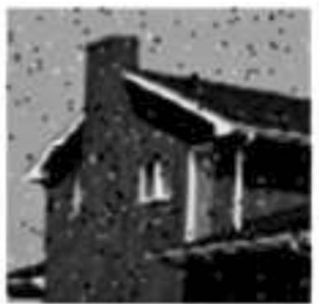

$\delta=0,04$

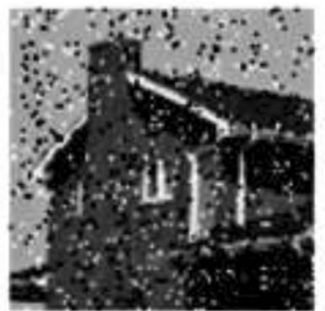

$\delta=0.09$

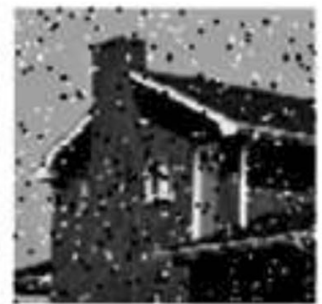

$\delta=0.05$

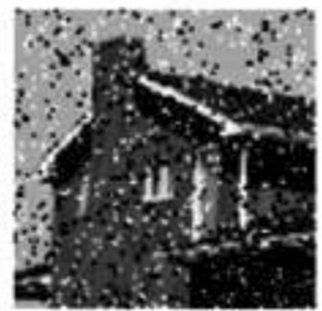

$\delta=0.10$

Fig. 7 House image that contains pepper and salt noises with different intensities 


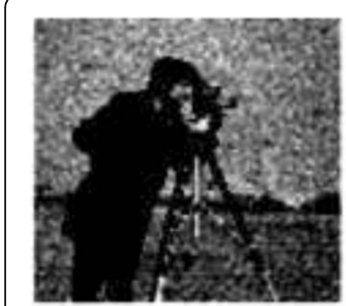

$\sigma^{2}=0.01$

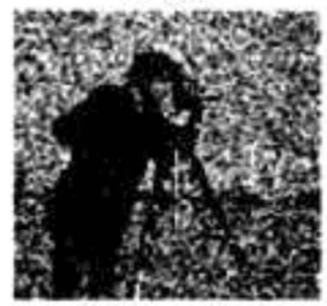

$\sigma^{2}=0.06$

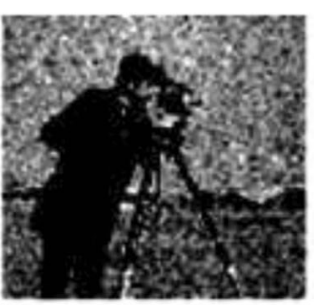

$\sigma^{2}=0.02$

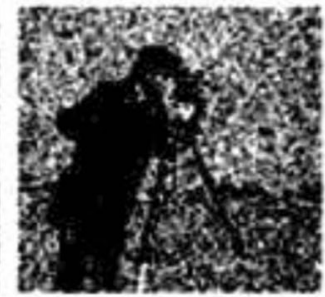

$\sigma^{2}=0.07$

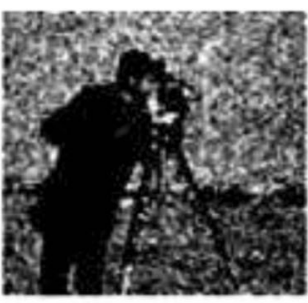

$\sigma^{2}=0.03$

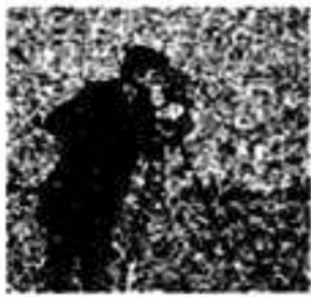

$\sigma^{2}=0.08$
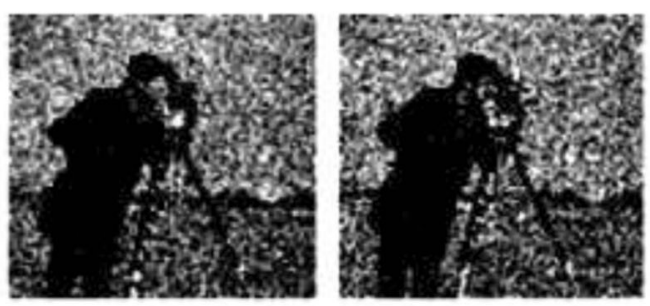

$\sigma^{2}=0.04$

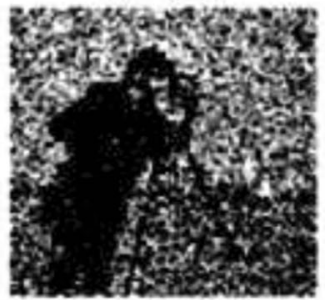

$\sigma^{2}=0.09$

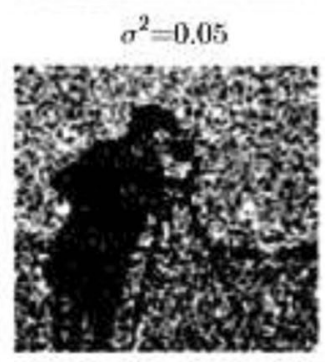

$\sigma^{2}=0.10$

Fig. 8 Cameraman image that contains Gaussian noise with different variances

is used to combine the MS graph with the PAN image, and the fusion image is shown in Fig. 6 with the AWNMMOA algorithm.

To test the noise resistance robustness of each algorithm, the house image as shown in Fig. 7 and the cameraman image as shown in Fig. 8 are selected as the test image, in which the value $0.01,0.02$, and 0.10 are taken for the intensity $\delta$ of the salt and pepper noise in the house image and the Gaussian noise variance $\sigma^{2}$ in the cameraman image in turn. From Figs. 9 and 10, it can be seen that with the constant increase in the noise intensity, the regional consistency of each algorithm gradually decreases, and the pixel misclassification rate gradually increases. The noise resistance performance of the traditional three-dimensional Otsu method and the three-dimensional Otsu algorithm based on decomposition is relatively poor due to the application of the direct finite difference method for the division of the histogram. And the synthesis performance rapidly decreases with the increase of the noise intensity. The multi-objective optimization algorithm and the Otsu method for the multi-objective cross section method of the adaptive wireless network put forward in this paper are both based on the image projection histogram and then adopting the post-processing strategy to correct the noise, which have relatively strong noise resistance robustness. In this paper, the multi-objective cross section Otsu method for the adaptive wireless network takes the multi-objective optimization threshold value $Q$ of the adaptive wireless network as a variable. The maximum between-class variance and the signal to noise

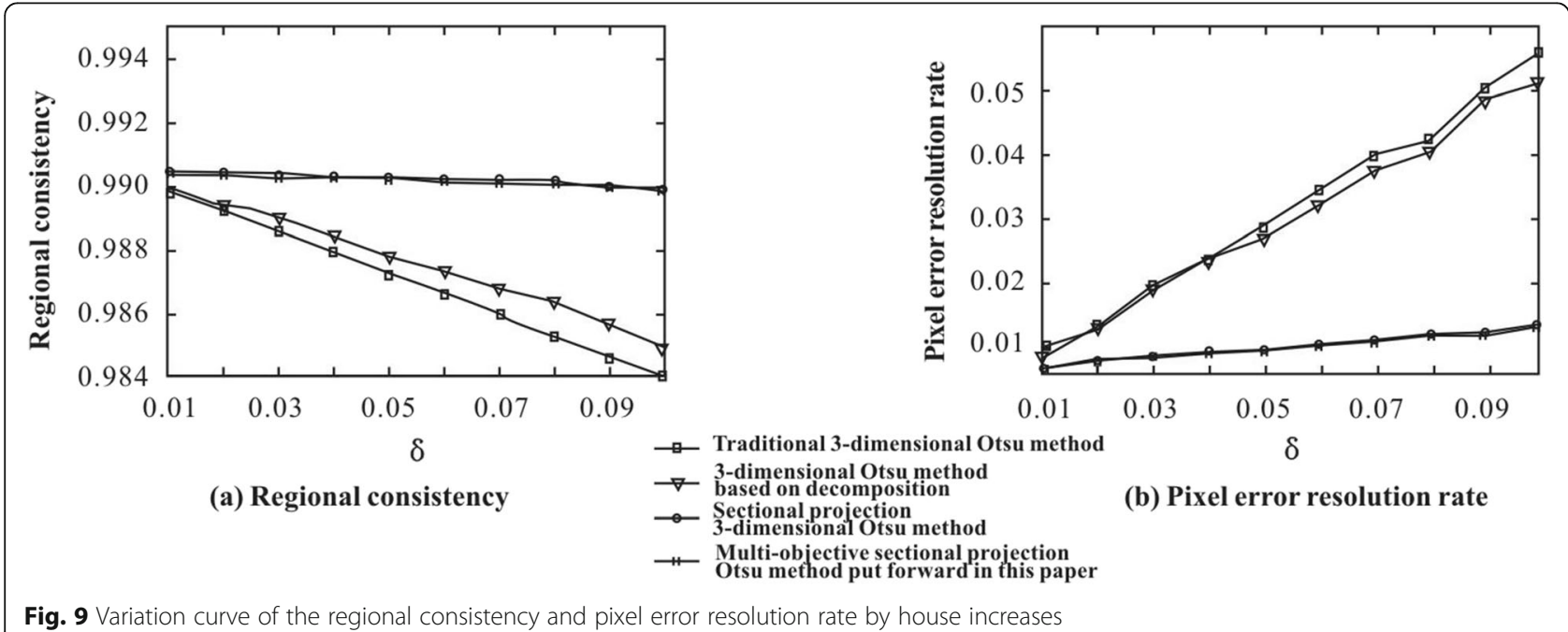

Fig. 9 Variation curve of the regional consistency and pixel error resolution rate by house increases 


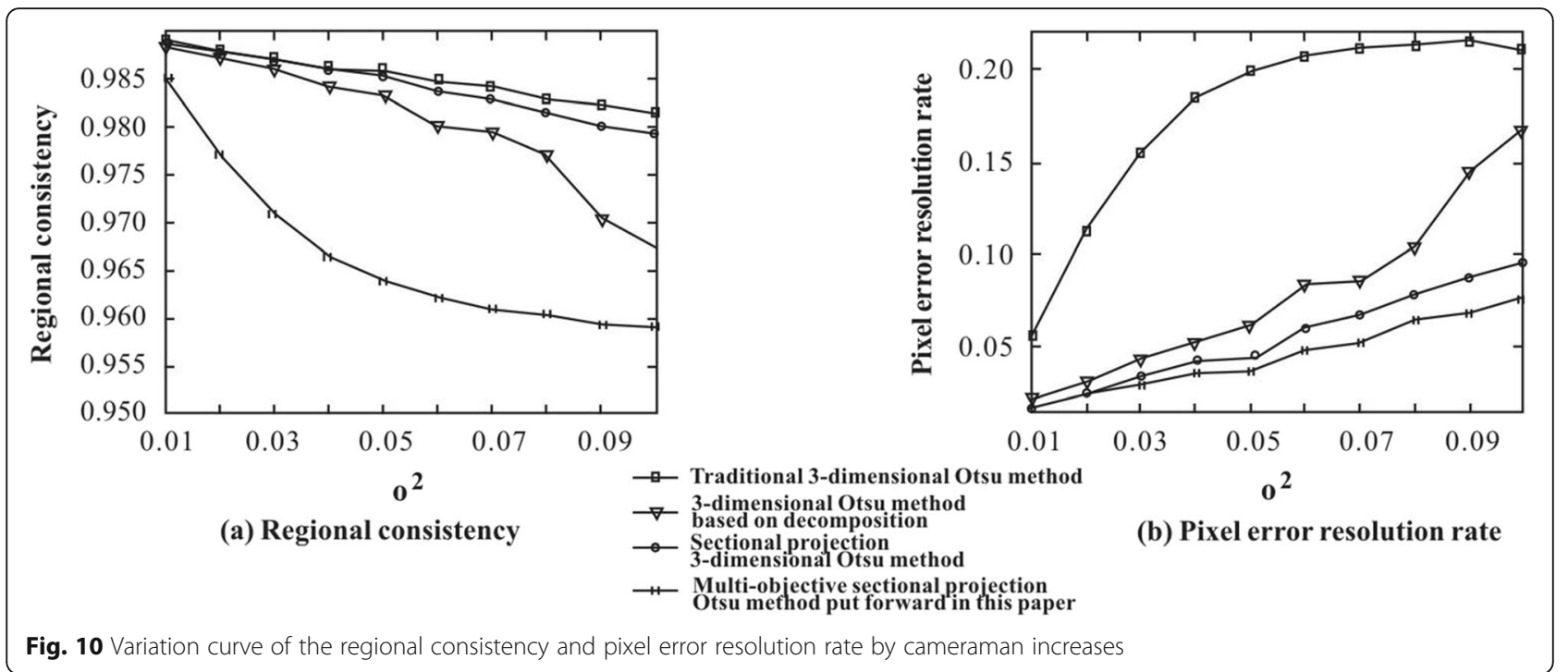

ratio criteria are used to determine the optimal threshold value $T$ and the adaptive threshold value $Q$. It can take the accuracy of the image synthesis and the noise immunity into consideration at the same time. Therefore, it has stronger adaptability to the synthesis of images with different noise conditions. It shows that the algorithm is effective and robust and proves the feasibility of the method.

\section{Conclusions}

To enhance the universality of the multi-objective optimization algorithm and better synthesize the noise image, a kind of adaptive wireless network multi-objective optimization algorithm based on the image synthesis is put forward in this paper. In this method, the threshold value $Q$ in the post-processing of the multi-objective optimization algorithm is regarded as the additional variable. And the multi-objective optimization algorithm for the adaptive wireless network is established by using the two criteria of the maximum between-class variance and the signal to noise ratio, so that the algorithm can take the accuracy of the image synthesis and noise immunity performance into consideration at the same time. The comparison experiment on the image synthesis with the other optimization algorithms shows that the algorithm put forward in this paper has obvious advantages in the convergence accuracy, the algorithm robustness, and other aspects. In this paper, the multi-objective optimization algorithm of the adaptive wireless network has achieved good synthesis effect for the images with different types of noise, which has effectively verified the effectiveness of the algorithm. The robustness test shows that with the constant increase in the noise intensity, the synthetic performance of the multi-objective optimization algorithm for the adaptive wireless network put forward in this paper is good and relatively stable. In view of all the results, although the efficiency of the method put forward in this paper is slightly reduced, the algorithm has strong noise resistance performance and good robustness.

\section{Abbreviation}

AWNMMOA: Adaptive wireless network multi-objective optimization algorithm; MOA: Multi-objective optimization algorithm; PSNR: Peak signal to noise ratio

\section{Acknowledgements}

The authors thank the editor and anonymous reviewers for their helpful comments and valuable suggestions.

\section{About the authors}

Zhang Jianwei is a Master of Communication and Information System and a lecturer. In 2015, he graduated from Xi'an Electronic and Science University and worked in Baoji University of Arts and Sciences. His research interests include network architecture and network information security.

Zhang Xueya is a Master of Computer Science and a lecturer. In 2013, she graduated from Xi'an Technological University and worked in Baoji University of Arts and Sciences. Her research interests include big data analysis and cloud computing.

\section{Funding}

This work was supported in part by a grant from the National Natural Science Project (11675001), Shaanxi Provincial Education Department scientific research project (16JK1045), Baoji Science and Technology project (2013R1-5,14GYGG-4), and Key project of Baoji University of Arts and Sciences (ZK2017002).

Availability of data and materials

We can provide the data.

\section{Authors' contributions}

JZ conceived the original idea for the project and improved the algorithm; the first version of the paper is written by him. XZ confirmed the performance of the algorithm. All authors contributed to the helpful discussions and analyzed the results. Both authors read and approved the final manuscript. 
Ethics approval and consent to participate

Not applicable.

\section{Consent for publication}

Not applicable.

\section{Competing interests}

The authors declare that they have no competing interests

\section{Publisher's Note}

Springer Nature remains neutral with regard to jurisdictional claims in published maps and institutional affiliations.

\section{Author details}

${ }^{1}$ College of Physics and Optoelectronic Technology, Baoji University of Arts and Sciences, Baoji 721016, China. ${ }^{2}$ College of Computer Science, Baoji University of Arts and Sciences, Baoji 721016, China.

Received: 26 April 2018 Accepted: 13 June 2018

Published online: 26 June 2018

\section{References}

1. R Devarajan, SC Jha, U Phuyal, VK Bhargava, Energy-aware resource allocation for cooperative cellular network using multi-objective optimization approach. IEEE Trans. Wirel. Commun. 11(5), 1797-1807 (2012)

2. Y Sun, DWK Ng, J Zhu, R Schober, Multi-objective optimization for robust power efficient and secure full-duplex wireless communication systems. IEEE Trans. Wirel. Commun. 15(8), 5511-5526 (2016)

3. YF Li, G Sansavini, E Zio, Non-dominated sorting binary differential evolution for the multi-objective optimization of cascading failures protection in complex networks. Reliab. Eng. Syst. Saf. 111(1), 195-205 (2013)

4. S Fazlollahi, G Becker, F Maréchal, Multi-objectives, multi-period optimization of district energy systems: iii-distribution networks. Comput. Chem. Eng. 66(4), 82-97 (2014)

5. GF Porzio, G Nastasi, V Colla, M Vannucci, TA Branca, Comparison of multiobjective optimization techniques applied to off-gas management within an integrated steelwork. Appl. Energy 136, 1085-1097 (2014)

6. N Liu, D Plets, SK Goudos, L Martens, W Joseph, Multi-objective network planning optimization algorithm: human exposure, power consumption, cost, and capacity. Wirel. Netw 21(3), 841-857 (2015)

7. Z Sun, Y Zhang, Y Nie, W Wei, J Lloret, H Song, Casmoc: a novel complex alliance strategy with multi-objective optimization of coverage in wireless sensor networks. Wirel. Netw 23(4), 1201-1222 (2017)

8. JJ Deng, CHC Leung, Dynamic time warping for music retrieval using time series modeling of musical emotions. IEEE Trans. Affect. Comput. 6(2), 137-151 (2015)

9. EA Khalil, S Ozdemir, Reliable and energy efficient topology control in probabilistic wireless sensor networks via multi-objective optimization. J. Supercomput. 73(6), 1-25 (2016)

10. Q Wu, H Ren, W Gao, J Ren, Multi-objective optimization of a distributed energy network integrated with heating interchange. Energy 109, 353-364 (2016)

\section{Submit your manuscript to a SpringerOpen ${ }^{\circ}$ journal and benefit from:}

- Convenient online submission

- Rigorous peer review

- Open access: articles freely available online

- High visibility within the field

- Retaining the copyright to your article 